\title{
RECUPERAÇÃO DE CROMO PRESENTE EM POEIRAS E LAMAS PROVENIENTES DA FABRICAÇÃO DE AÇO INOXIDÁVEL - INFLUÊNCIA DA BASICIDADE
}

Pedro José Nolasco Sobrinho ${ }^{1}$ Jorge Alberto Soares Tenório ${ }^{2}$

\section{Resumo}

Este trabalho apresenta o estudo da influência da basicidade da escória gerada no processo de redução silicotérmica do óxido de cromo presente em resíduos siderúrgicos do processo de fabricação de aço inoxidável. Foram testadas as basicidades 0,5, I e I,5. Para a realização do estudo, dois diferentes resíduos siderúrgicos de indústrias brasileiras foram testados, uma poeira (PM) e uma lama(LV). Experimentos de introdução de briquetes de resíduos com alto teor de cromo em aço líquido $(1020)$ foram realizados na temperatura de $1.600^{\circ} \mathrm{C}$. Periodicamente foram recolhidas amostras de aço do banho para a determinação do teor de cromo das amostras. Um equipamento experimental, em escala de laboratório, composto por um forno elétrico foi utilizado para a fusão dos reagentes (aço e briquete). Em função do resultado da análise química foram calculadas as frações de redução do óxido de cromo dos briquetes testados. Os resultados mostraram que a redução do óxido de cromo é favorecida para valores de basicidade superiores à unidade e que o teor de alumina da escória não influenciou o processo de redução da cromita do resíduo.

Palavras-chave: Resíduo siderúrgico; Cromo; Poeira; Lama; Aciaria.

\section{CHROMIUM RECOVERY FROM STAINLESS STEELMAKING DUSTS AND SLUDGES - IMPORTANCE OF THE BASICITY}

\begin{abstract}
In this study two different wastes generated during Brazilian stainless steelmaking were tested. They were agglomerated in the briquette form mixed with $\mathrm{FeSi} 75$ and $\mathrm{CaO}$. The $\mathrm{CaO}$ was added to adjust the final basicity respectively to $0.5, \mathrm{I}$ and I.5. Then six kinds of briquettes were performed and used. Tests were carried out in a laboratory piece of equipment where 1020 steel was melted at $1,600^{\circ} \mathrm{C}$ under inert atmosphere. In each test one briquette of waste was introduced in liquid steel and steel samples were withdrawn periodically. The results shown that the chromium oxide reduction occurs at basicity equal or greater than one despite of the alumina content in the slag.
\end{abstract}

Key-words: Wastes; Chromium; Dust; Sludge; Steel plant.

\section{INTRODUÇÃO}

A basicidade é uma ferramenta largamente utilizada pelos aciaristas para se avaliar a extensão das reações químicas que ocorrem durante o processo de refino do aço, pois a escória no estado líquido pode ser considerada uma solução iônica. Baseado neste fato pode-se considerar que a basicidade de um óxido MO é determinada pela eletronegatividade do metal $M$, ou seja, a medida da facilidade na qual um elétron pode ser removido do átomo para formar um íon. ${ }^{(1)}$

A eletronegatividade de $M$ é que determina a natureza da ligação entre o metal e o oxigênio no óxido. Sendo assim, ela é determinante de muitas propriedades físico-químicas da escória, onde os óxidos básicos são aqueles formados a partir de elementos mais eletropositivos como o carbono e o enxofre. (1)

A formação dos óxidos básicos envolve a transferência de elétrons do metal para o oxigênio para formar cátions de metal e ânions oxigênio, os quais são ligados no óxido por forças eletrostáticas. As cargas positivas dos cátions são rodeadas pelas cargas negativas dos ânions, e vice-versa, formando uma estrutura de cristal simples.

As formas encontradas para se avaliar o fenômeno que envolve a basicidade de uma escória e seu processo de dissolução variaram em

\footnotetext{
' Sócio titular da ABM. Pós-Doutor, Doutor em Engenharia(PMT-Poli USP). Área: Engenharia Metalúrgica e de Materiais. M.Sc. em Engenharia de Materiais(REDEMAT). Engenheiro Metalurgista(UFOP). Gerente de Pesquisa, Desenvolvimento e Controle de Qualidade da VAMTEC S/A. Centro de Pesquisa e Desenvolvimento da VAMTEC - CPV. Rua 5, $n^{\circ}$ 6, Alegre - 35I8I-0I0 - Timóteo - MG.

${ }^{2}$ Sócio titular da ABM Professor Titular. Departamento de Engenharia Metalúrgica e de Materiais da Escola Politécnica da USP. Av. Prof. Mello Moraes. 2464 - 05508-900 - Sâo Paulo - SP
} 
função da evolução do estudo de seu comportamento físico-químico. As mais simples em concepção, porém de alta praticidade, são as relações que envolvem o percentual em massa dos óxidos básicos pelos ácidos. Para o cálculo da basicidade binária $\left(B_{b}\right)$ de uma escória, usa-se a seguinte relação:

$$
\mathrm{B}_{\mathrm{b}}=\frac{\% \mathrm{CaO}}{\% \mathrm{SiO}_{2}}
$$

A basicidade também pode ser definida como sendo:

$$
\mathrm{B}_{\mathrm{t}}=\frac{\%(\mathrm{CaO}+\mathrm{MgO})}{\%\left(\mathrm{SiO}_{2}\right)}
$$

Esta relação é conhecida como basicidade ternária $\left(B_{t}\right)$.

$\mathrm{Na}$ evolução do cálculo da basicidade foi feita a introdução de dois importantes óxidos:

- MgO, óxido básico, geralmente encontrado no revestimento do refratário; e

- $\mathrm{Al}_{2} \mathrm{O}_{3}$, óxido de caráter anfótero, aqui considerado como ácido, podendo ser proveniente do refratário ou na forma de produto da desoxidação. Assim, a relação para o cálculo da chamada basicidade quaternária $\left(\mathrm{B}_{q}\right)^{(1,2)}$ é:

$$
\mathrm{B}_{\mathrm{q}}=\frac{\%(\mathrm{CaO}+\mathrm{MgO})}{\%\left(\mathrm{SiO}_{2}+\mathrm{Al}_{2} \mathrm{O}_{3}\right)}
$$

Porém, uma deficiência encontrada nestes tipos de relações é que elas envolvem decisões arbitrárias quanto à natureza ácida ou básica de um óxido. Para os casos em que ocorre a adição de fluorita na formação de escória, ou com a presença de óxidos de caráter anfótero, como $\circ \mathrm{Al}_{2} \mathrm{O}_{3}$ e $\circ \mathrm{TiO}_{2}$, as relações acima não exprimem a realidade.

Buscando suprir estes problemas surgiu o conceito de basicidade ótica, onde a basicidade é enfocada em termos da capacidade de doação de elétrons. A palavra ótica deriva da possibilidade de se medir a basicidade da escória espectroscopicamente. ${ }^{(1,2)}$

Após uma série de ensaios ${ }^{(1,2)}$ foi determinado que a basicidade ótica de um óxido $(\Lambda)$ é função da eletronegatividade de Pauling $(X)$, conforme a relação a seguir:

$$
\frac{1}{\Lambda}=1,36(\mathrm{X}-0,26)
$$

Durante a produção de aço inoxidável, o elemento silício, geralmente, é utilizado na forma de liga $\mathrm{FeSi}$, para reduzir as escórias com alto teor de óxido de cromo.

Outros metais como o alumínio e o titânio também poderiam ser utilizados como redutores, mas poderiam formar inclusões no aço. ${ }^{(3)}$ Considerando-se que o cromo presente na escória esteja na forma $\mathrm{Cr}_{3} \mathrm{O}_{4(1)}$, a sua redução através do silício pode ser representada como sendo: ${ }^{(3-5)}$

$$
\mathrm{Cr}_{3} \mathrm{O}_{4(1)}+2 \underline{\mathrm{Si}}=3 \underline{\mathrm{Cr}}+2 \mathrm{SiO}_{2(1)}
$$

Da termodinâmica clássica, sabe-se que:

$$
\Delta \mathrm{G}^{\mathrm{O}}=\Delta \mathrm{H}^{\mathrm{O}}-\mathrm{T} \Delta \mathrm{S}^{\mathrm{O}}
$$

As expressões para a energia livre padrão, $\Delta \mathrm{G}^{\circ}$, em função da temperatura e para a constante de equilíbrio, K, para a Reação (5), são, respectivamente:

$$
\begin{aligned}
& \Delta \mathrm{G}^{\mathrm{O}}=-113.302-28,2 \mathrm{~T}^{(5)} \mathrm{J} / \mathrm{Mol} \\
& \mathrm{K}=\frac{\mathrm{h}_{\mathrm{Cr}^{3}}{ }^{3} \cdot \mathrm{a}_{\mathrm{SiO}_{2}}{ }^{2}}{\mathrm{a}_{\mathrm{Cr}_{3} \mathrm{O}_{4}} \cdot \mathrm{h}_{\mathrm{Si}}{ }^{2}}
\end{aligned}
$$

onde $\mathrm{h}$, representa atividade henriana e a, atividade raoultiana.

Assim, $\mathrm{h}_{\mathrm{cr}}=$ atividade henriana do cromo; $\mathrm{h}_{\mathrm{si}}=$ atividade henriana do silício; $\mathrm{a}_{\mathrm{SiO}_{2}}=$ atividade raoultiana da sílica; e $\mathrm{a}_{\mathrm{Cr}_{3} \mathrm{O}_{4}}=$ atividade raoultiana da cromita.

A reação de redução do cromo pelo silício pode ser aproximada para:

$$
\mathrm{Cr}_{2} \mathrm{O}_{3(1)}+1,5 \underline{\mathrm{Si}}=2 \underline{\mathrm{Cr}}+1,5 \mathrm{SiO}_{2(1)}
$$

Analogamente ao tratamento realizado para a Reação (5) Pode-se desenvolver as expressões para a energia livre padrão, $\Delta \mathrm{G}^{\circ}$, em função da temperatura e para a constante de equilíbrio, $\mathrm{K}$ :

$$
\begin{aligned}
& \Delta \mathrm{G}^{\mathrm{O}}=-192.984+35,3 \mathrm{~T} \mathrm{~J} / \mathrm{mol} \\
& \mathrm{K}=\frac{\mathrm{h}_{\mathrm{Cr}^{2} \cdot \mathrm{a}_{\mathrm{SiO}_{2}}}{ }^{1.5}}{\mathrm{~h}_{\mathrm{Si}}{ }^{1.5} \cdot \mathrm{a}_{\mathrm{Cr}_{2} \mathrm{O}_{3}}}
\end{aligned}
$$

As Reações (5) e (9) podem ser consideradas como reações que consistem de duas etapas simultâneas onde, numa delas, o potencial de oxigênio do banho é diminuído pela adição do silício e na outra o cromo é transferido da escória para $\circ$ metal para que haja $\circ$ restabelecimento do equilíbrio da reação no novo potencial de oxigênio.

Nesta etapa do processo, para aumentar a eficiência do processo e proteger o refratário do forno, pode-se diminuir a temperatura do banho antes da adição da liga $\mathrm{FeSi}$, uma vez que as Reações (5) e (9) são exotérmicas.

Além da utilização do silício durante a recuperação de óxido de cromo contido na escória, é necessário atuar na basicidade da mesma para que se tenha um favorecimento na recuperação do cromo ali contido. A recuperação deste elemento é favorecida pela alta basicidade e alto teor de silício no aço.

A Figura I mostra $\circ$ efeito destas duas variáveis na recuperação do cromo.

A razão da influência da basicidade pode ser entendida avaliando a constante de equilíbrio da Reação (9): ${ }^{(5)}$ 


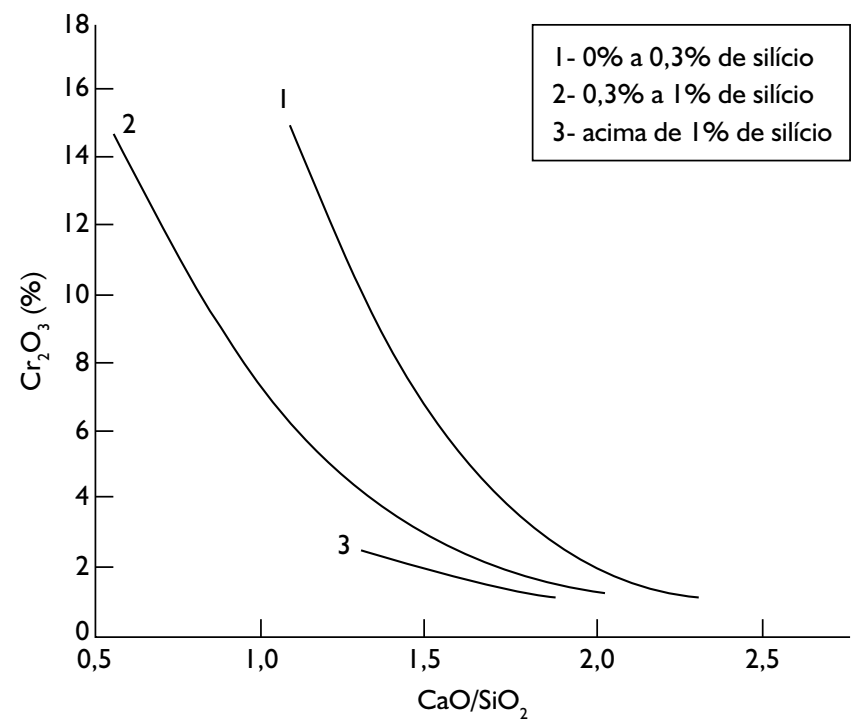

Figura I. Efeito da basicidade binária e do teor de silício na redução do $\mathrm{Cr}_{2} \mathrm{O}_{3}{ }^{(4)}$

Quando a escória está saturada com o óxido de cromo a relação $\frac{h_{C r}}{h_{S i}}$ é inversamente proporcional à atividade da sílica na escória. Assim, para um dado valor de $\mathrm{h}_{\mathrm{Si}}$ a recuperação do cromo aumenta quando a basicidade da escória aumenta.

Do ponto de vista termodinâmico, foi verificado que a recuperação do cromo oxidado na escória é favorecida por: ${ }^{(6)}$

- baixas temperaturas;

- baixos teores de cromo no banho;

- altos teores de silício no banho; e

- altas basicidades de escória.

Visando ao entendimento da influência da basicidade sobre o processo de redução do óxido de cromo presente em resíduos siderúrgicos foi realizado este estudo, onde se fez a adição de briquetes de resíduos provenientes da fabricação de aço inoxidável em aço líquido na temperatura de $1.600^{\circ} \mathrm{C}$, utilizando-se um equipamento experimental composto por um forno vertical. Três diferentes valores de basicidade binária da escória final formada no processo foram selecionadas para o estudo, 0,5; I e I,5. Periodicamente, amostras de aço do banho foram coletadas para análise do teor de cromo, assim como a escória final para identificação das fases presentes.

\section{METODOLOGIA}

Neste estudo foram utilizados dois diferentes tipos de resíduos, contendo óxido de cromo, oriundos da produção de aço inoxidável no Brasil.

Identificados como PM e LV foram, inicialmente, caracterizados utilizando-se das técnicas de análise química, análise granulométrica, picnometria, difração de raios $\mathrm{X}$, microscopia eletrônica de varredura (MEV) e análise de micro-regiões com EDS.

Depois, separadamente, os resíduos foram aglomerados na forma de briquetes, juntamente com o redutor, a liga FeSi75, e cal para o ajuste da basicidade. $O$ cálculo da quantidade necessária de redutor para a redução dos óxidos dos resíduos foi feito com base na análise química dos mesmos. A quantidade de cal utilizada em cada briquete para o ajuste da basicidade foi calculada considerando-se a quantidade de sílica formada durante a reação de redução dos óxidos do resíduo. Foram fabricados seis briquetes para os testes no equipamento experimental constituído de um forno vertical. Foram utilizadas basicidades 0,5, I e I,5 na elaboração da composição dos briquetes.

Então, seis experimentos de adição de briquete em aço líquido foram realizados, sendo três com cada tipo de resíduo e, periodicamente, foram retiradas amostras do banho para a análise do teor de cromo.

Cada experimento de fusão do briquete em aço líquido envolveu os seguintes passos:

- introdução do cadinho de alumina contendo aço no equipamento experimental;

- acionamento do forno e do controlador para a realização do experimento a uma taxa de aquecimento de $10^{\circ} \mathrm{C}$ por minuto até a temperatura de ensaio $\left(1.600^{\circ} \mathrm{C}\right)$ e manutenção do equipamento nesta temperatura por I,5 h;

- abertura do sistema de entrada de gás argônio injetado pela parte inferior do dispositivo, através de tubos de aço inoxidável a uma vazão, controlada por um rotâmetro, de 5 L/minuto, com o intuito de controlar a atmosfera onde o aço era fundido.

- retirada de uma amostra de aço líquido, na temperatura estabelecida

- adição do briquete de resíduos siderúrgicos no aço líquido;

- retirada periódica de amostras do banho;

- resfriamento do banho à mesma velocidade utilizada para o aquecimento;

- fechamento do sistema de injeção de gases inertes, após a solidificação do aço e da escória formada durante o experimento;

- retirada do cadinho do equipamento, retirada de uma amostra de escória; e

- desligamento do forno e do controlador.

Um termopar tipo B monitorava a temperatura dos experimentos, durante os quais não se notou nenhuma alteração brusca da temperatura.

As amostras de aço foram coletadas introduzindo-se um amostrador de quartzo à vácuo a cerca de $25 \mathrm{~mm}$ de profundidade, retirando-o após o preenchimento do mesmo. $\mathrm{O}$ amostrador 
cilíndrico possuía, em média, $132 \mathrm{~mm}$ de comprimento, $4 \mathrm{~mm}$ de diâmetro e uma massa média de $6,07 \mathrm{~g}$.

Cada experimento durou:

- 2,5 h para atingir $1.500^{\circ} \mathrm{C}$;

- I,5 h para atingir $1.600^{\circ} \mathrm{C}$;

- I,5 h para a estabilização da temperatura do sistema a I.600 $\mathrm{C}$;

- I,0 h para adição do briquete de resíduos no aço líquido, retirada de amostras do banho e preparo das amostras para envio à análise química; e

- I,5 h para abaixamento da temperatura do banho até a sua solidificação, totalizando $8 \mathrm{~h}$.

A Tabela I apresenta as condições experimentais deste estudo. As amostras foram retiradas do banho em intervalos de três minutos, sendo seis de cada experimento, totalizando 36 amostras que tiveram seus teores de cromo analisados.

Com relação à primeira coluna da Tabela $\mathrm{I}$, as duas primeiras letras identificam o tipo de resíduo utilizado e os quatro números seguintes representam a temperatura do ensaio. A letra minúscula

Tabela I. Condições experimentais.

\begin{tabular}{|c|c|c|c|c|c|}
\hline \multirow[b]{2}{*}{ Experimento } & \multicolumn{3}{|c|}{$\begin{array}{c}\text { Composição do briquete } \\
\text { (\% em massa) }\end{array}$} & \multirow[b]{2}{*}{$\begin{array}{c}\text { Massa de } \\
\text { aço (g) } 1020\end{array}$} & \multirow[b]{2}{*}{$\begin{array}{c}\text { Massa de } \\
\text { briquete (g) }\end{array}$} \\
\hline & Resíduo & FeSi75 & Cal & & \\
\hline PMI600b0.5 & 75,6 & 24,4 & - & 406,24 & 30,20 \\
\hline PMI600bl.0 & 74,6 & 24,1 & $\mathrm{I}, 3$ & 405,47 & 30,39 \\
\hline PMI600bI.5 & 73,7 & 23,8 & 2,5 & 406,66 & 29,91 \\
\hline LVI600b0.5 & 78,1 & 21,5 & 0,4 & 408,27 & 30,11 \\
\hline LVI600bI.0 & 77,5 & 21,3 & $\mathrm{I}, 2$ & 408,07 & 29,81 \\
\hline LVI600bI.5 & 77,1 & 21,2 & $\mathrm{I}, 7$ & 405,37 & 29,72 \\
\hline
\end{tabular}

seguida de um número identifica a basicidade da escória que seria formada na introdução do briquete no aço líquido.

A técnica de difração de raios $X$ foi utilizada para a identificação das fases mais prováveis presentes em duas amostras de escórias retiradas após a solidificação do aço em dois experimentos realizados (PMI600b0.5 e PMI600bl.0). Foi utilizado o software $X$-Pert da Phillips na identificação das fases presentes.

Utilizou-se a técnica de difração de raios $X$ juntamente com o software X-Pert da Phillips para a identificação das fases mais prováveis presentes em duas amostras de escórias retiradas após a solidificação do aço em dois experimentos realizados (PMI600b0.5 e PMI600bl.0).

\section{RESULTADOS E DISCUSSÃO}

\section{I Caracterização dos Resíduos}

Através da caracterização, constatou-se que os resíduos $\mathrm{PM}$ e LV são constituídos predominantemente de partículas esféricas, com diâmetro médio de $1,7 \mu \mathrm{m}$ e $4,0 \mu \mathrm{m}$, respectivamente. O resíduo PM apresentou densidade real igual a $3,5 \mathrm{~g} / \mathrm{cm}^{3}$ e o resíduo $L V, 4,9 \mathrm{~g} / \mathrm{cm}^{3}$.

A principal fase encontrada nos resíduos foi a fase $\mathrm{FeO}$. $\mathrm{Cr}_{2} \mathrm{O}_{3}$.

Tecnologia em Metalurgia e Materiais, São Paulo, v.4, n.2, p. 50-55, out.-dez. 2007
A composição química dos resíduos é a apresentada na Tabela 2.

Resultado mais detalhado referente à caracterização dos resíduos deste estudo pode ser encontrado na literatura em trabalhos já publicados. ${ }^{(7-9)}$

Tabela 2. Composição química dos resíduos.

\begin{tabular}{lrr}
\hline \multicolumn{3}{c}{ Composição química (\% em massa) } \\
\cline { 2 - 3 } Elemento & \multicolumn{2}{c}{ Resíduo } \\
\hline $\mathrm{Ca}$ & $\mathbf{P M}$ & $\mathbf{L V}$ \\
$\mathrm{Cr}$ & $\mathrm{I}, 20$ & 0,38 \\
$\mathrm{Fe}_{\text {total }}$ & 7,00 & 13,40 \\
$\mathrm{Fe}_{\text {metálico }}$ & 53,50 & 51,30 \\
$\mathrm{~K}$ & 0,30 & 0,10 \\
$\mathrm{Na}$ & 3,20 & 2,66 \\
$\mathrm{Ni}$ & 1,90 & 2,05 \\
$\mathrm{P}$ & 2,56 & 2,93 \\
$\mathrm{~Pb}$ & 0,82 & 0,03 \\
$\mathrm{Mg}$ & 0,03 & 0,15 \\
$\mathrm{Si}$ & 0,04 & 0,05 \\
$\mathrm{Zn}$ & $\mathrm{I}, 68$ & 0,93 \\
\hline
\end{tabular}

\subsection{Experimentos de Fusão dos Briquetes de Resíduo Siderúrgico}

A Tabela 3 e a Figura 2 apresentam os resultados obtidos para a fração de redução do óxido de cromo dos resíduos PM e LV nos experimentos realizados com diferentes basicidades. $A$ fração de redução do óxido de cromo foi calculada com base nos resultados da análise química realizada nas amostras retiradas do banho e na composição química dos componentes, através da seguinte relação:

$$
\mathrm{f}_{\mathrm{r}}=\frac{\mathrm{M}_{\mathrm{i}}-\mathrm{M}_{\mathrm{t}}}{\mathrm{M}_{\mathrm{i}}}
$$

onde $M_{i}$ representa a massa inicial do óxido do metal no briquete; $M_{t}$ representa a massa do óxido do metal no briquete em um instante $t$ após o início do experimento.

Pela Figura 2 pode-se observar que a basicidade da escória influenciou a redução do cromo, principalmente nos primeiros minutos do experimento. Nota-se que os menores valores de incorporação de cromo foram obtidos na basicidade de 0,5. Quando se aumentou a basicidade da escória para I e I,5 houve um aumento na redução do óxido de cromo. Uma maior fração de redução significa uma maior incorporação de cromo na liga. Observa-se que até os três primeiros minutos pelo menos $75 \%$ do óxido de cromo presente nos resí- 
Tabela 3. Influência da basicidade na redução do óxido de cromo dos resíduos.

\begin{tabular}{|c|c|c|c|c|c|c|}
\hline \multirow{3}{*}{$\begin{array}{c}\text { Tempo de } \\
\text { retirada da amostra } \\
\text { do banho (minuto) }\end{array}$} & \multicolumn{6}{|c|}{ Frações de redução do óxido de cromo (\%) } \\
\hline & \multicolumn{2}{|c|}{$B_{b}=0,5$} & \multicolumn{2}{|c|}{$B_{b}=I, 0$} & \multicolumn{2}{|c|}{$B_{b}=I, 5$} \\
\hline & PM & LV & PM & $\mathbf{L V}$ & PM & $\mathbf{L V}$ \\
\hline 3 & 0,75 & 0,80 & 0,82 & 0,86 & 0,80 & 0,86 \\
\hline 6 & 0,82 & 0,87 & 0,85 & 0,90 & 0,96 & 0,92 \\
\hline 9 & 0,89 & 0,93 & 0,94 & 0,96 & 0,97 & 0,97 \\
\hline 12 & 0,93 & 0,94 & 0,95 & 0,96 & 0,97 & 0,98 \\
\hline 15 & 0,95 & 0,96 & 0,97 & 0,97 & 0,98 & 0,98 \\
\hline 18 & 0,95 & 0,96 & 0,98 & 0,97 & 0,98 & 0,98 \\
\hline
\end{tabular}

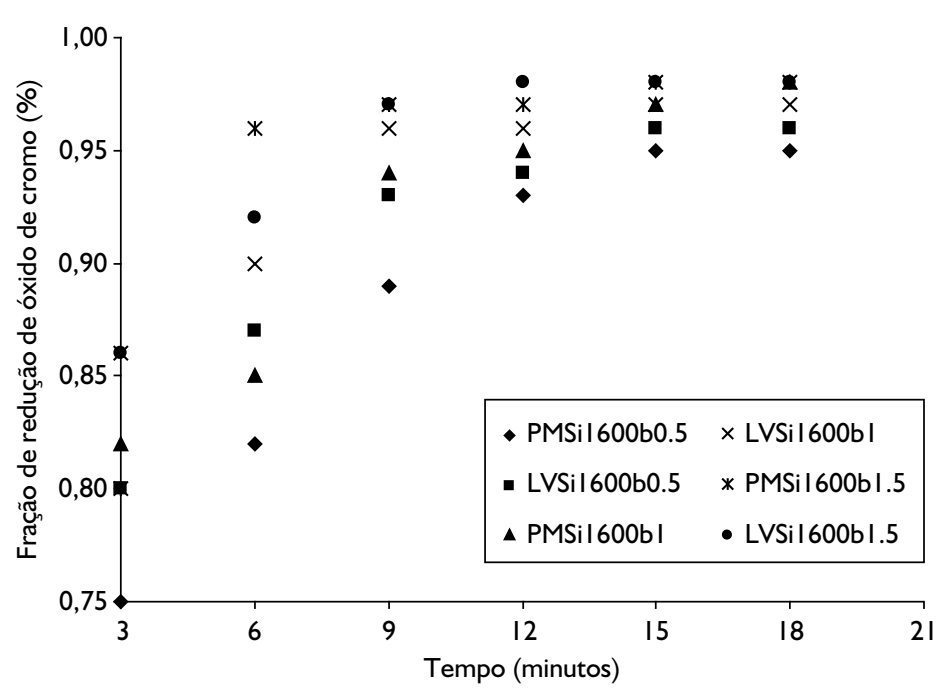

Figura 2. Redução do óxido de cromo dos briquetes de resíduos siderúrgicos.

duos havia sido reduzido. Isto mostra que o processo de redução ocorre muito rapidamente, concentrando-se nos primeiros minutos de contato entre o resíduo e o aço líquido.

A redução do óxido de cromo dos resíduos siderúrgicos prosseguiu e, no tempo de 15 minutos, o sistema encontrava-se num estado próximo ao equilíbrio, onde a redução do óxido de cromo havia atingido pelo menos $95 \%\left(B_{b}=0,5\right)$. Para as basicidades de I e I,5 a redução do óxido de cromo é praticamente total.

Görnerup e Lahiri ${ }^{(10)}$ verificaram que na basicidade de I,5 ocorreram os valores máximos de redução de óxido de ferro e cromo.

Os resultados obtidos indicam que quando se aumenta a basicidade da escória a fração de redução de cromo aumenta e assim uma maior quantidade de óxido de cromo é reduzido, estando disponível para incorporação no aço. Tsomondo Simbi e Navara ${ }^{(I I)}$ verificaram a influência da basicidade da escória sobre a redução do óxido de cromo. Foi verificado no referido estudo que a redução do óxido de cromo é afetada com as mudanças da basicidade e que, independentemente de seu valor, até o intervalo de vinte minutos, a redução se processa mais rapidamente.

Nakasuga e colaboradores, ${ }^{(12)}$ que estudaram a redução carbotérmica de $\mathrm{Cr}_{2} \mathrm{O}_{3}$ pulverizado e do $\mathrm{Cr}_{2} \mathrm{O}_{3}$ presente em escória do sistema $\mathrm{CaO}-\mathrm{SiO}_{2}-\mathrm{Al}_{2} \mathrm{O}_{3}-\mathrm{CaF}_{2}$, na faixa de $1.300^{\circ} \mathrm{C}$ a $1.600^{\circ} \mathrm{C}$, verificaram que a $1.600^{\circ} \mathrm{C}$ o banho com escória de basicidade igual a I,I promoveu maior redução do óxido de cromo.

Kundrat e colaboradores verificaram em seus estudos de redução carbotérmica da cromitaa ${ }^{(13)}$ que para se obter um maior êxito na redução da mesma a basicidade ternária da escória deve ser ao menos igual a um, sendo desejável valores de basicidade de I,5 e 2,5. A alumina presente na escória, preferencialmente, deve situar-se no intervalo entre 15 e $25 \%$ e o teor de $\mathrm{MgO}$ da escória esteja compreendido entre $10 \%$ e $20 \%$ em massa. Um outro importante dado fornecido por Kundrat é que a relação $\frac{\mathrm{MgO}}{\mathrm{Al}_{2} \mathrm{O}_{3}}$ esteja na faixa de 0,3 a 0,8 .

No presente estudo foi verificado que a basicidade influencia a redução silicotérmica da cromita e que a redução ocorre mais rapidamente para $\circ$ valor de basicidade igual a I,5. Verificouse, ${ }^{(14)}$ também, que a redução silicotérmica da cromita presente em resíduos siderúrgicos, ocorreu, satisfatoriamente, nas basicidades de I e $\mathrm{I}, 5$, a $1.600^{\circ} \mathrm{C}$ e $1.635^{\circ} \mathrm{C}$, para um valor extremamente baixo(menor que 0,0I) da relação $\frac{\mathrm{MgO}}{\mathrm{Al}_{2} \mathrm{O}_{3}}$.

\subsection{Caracterização das Escórias Resultantes de Experimentos pela Técnica de Raios $X$}

Para o experimento PMI600b0.5 as fases mais prováveis identificadas foram: $\mathrm{Fe}_{2} \mathrm{SiO}_{4}$ (silicato de ferro), $\mathrm{FeCr}_{2} \mathrm{O}_{4}$ (cromita), $\mathrm{Ca}_{0,54} \mathrm{Fe}_{1,46} \mathrm{SiO}_{4}$ (silicato de cálcio e ferro), $\mathrm{MnAl}_{2} \mathrm{O}_{4}$ (galaxita) e $\mathrm{FeO}$ (wustita).

Para o experimento PMI600b a fase mais provável identificada foi a fase $\mathrm{CaAl}_{2} \mathrm{Si}_{2} \mathrm{O}_{8}$ (anortita).

A explicação para o surgimento das fases contendo alumina está no fato da utilização de cadinhos de alumina nos experimentos, já que na composição dos componentes não se tinha uma quantidade considerável deste elemento. $\mathrm{Na}$ temperatura dos experimentos, $1.600^{\circ} \mathrm{C}$, houve a transferência da alumina do cadinho para a escória, na linha da escória, implicando, assim, na sua contaminação até a saturação.

Observou-se que a escória do experimento PMI600b0.5 apresentou a fase cromita mostrando que nesta composição de briquete e basicidade a redução do óxido de cromo do resíduo não ocorreu completamente. Este fato também se repetiu para o óxido de ferro presente no resíduo. Uma provável explicação para a presença de cromita na escória do experimento PMI600b0.5 é que, devido à presença de uma maior quantidade de sílica na escória, tornando-a mais viscosa $\left(B_{b}=0,5\right)$, houve a dificuldade para a redução, movimentação e incorporação do cromo no aço, permanecendo parte do cromo reduzido na escória que, posteriormente, reoxidou-se na forma de cromita.

Nos experimentos realizados nas basicidades de I e I,5, como o experimento 
PMI600bI.0, não se identificou na escória nenhuma fase contendo cromo ou ferro. Foi identificada a fase anortita (óxidos de cálcio, silício e alumínio). Isto mostra que todo o óxido de cromo e ferro presente no resíduo antes do experimento foi reduzido, sendo estes completamente incorporados ao aço. Este fato confirma a influência benéfica do aumento da basicidade na redução e incorporação de óxido de cromo presente em resíduos siderúrgicos.

As escórias produzidas nos experimentos em que foram obtidos valores da fração de redução de cromo próximos à unidade, não possuíam ou possuíam em quantidades insignificantes, óxidos do elemento cromo na sua constituição, confirmando que nas basicidades I e I,5 a redução do óxido de cromo ocorre mais facilmente.

\section{CONCLUSÕES}

- A basicidade binária da escória formada no processo, durante a redução silicotérmica do óxido de cromo presente em resíduos siderúrgicos, influencia a redução, sendo que os valores de I e I,5 favoreceram a redução do óxido de cromo presente na escória e a sua incorporação à liga.

- A técnica de difração de raios X, utilizada na identificação das fases predominantes na escória final dos experimentos, confirmou que a redução de óxido de cromo presente em resíduo siderúrgico e a incorporação do cromo no aço ocorrem mais facilmente em basicidade binária entre I e I,5.

- A alumina proveniente do cadinho, que foi incorporada à escória durante o experimento, não influenciou o valor da fração de redução do óxido de cromo a $1600^{\circ} \mathrm{C}$.

\section{REFERÊNCIAS}

I BARRIOS, S.R.; Costa, E.A Interação físico-química de escórias sintéticas com o refratário de panelas de aço. In: SEMINARIO DE ACIARIA, REFRATARIOS, FORNOS ELETRICOS, FERRO-LIGAS E FUNDICAO, 25., 1993, Porto Alegre. Anais... São Paulo: ABM, 1993. p. 253-272.

2 BARRIOS, S.R.; COSTA, E. A.; FERREIRA, M. A. C.; AZEVEDO, F. R. S. Considerações metalúrgicas a respeito da utilização de escórias sintéticas em forno panela. In: SEMINÁRIO DE FUSÃO, REFINO E SOLIDIFICAÇÃO DOS AÇOS, 26. 1994, Salvador. Anais... São Paulo: ABM, 1994. p. 287-304.

3 ENGH, T.A. Stainless steelmaking in converter. In: Principles of metal refining. New York: Oxford University Press, 1992. p. 28I-30I.

4 TAYLOR, C.R. Electric furnace steelmaking. Warrendale: Iron and Steel Society, 1985. 395 p.

5 BODSWORTH, C.; BELL, H.B. Physical chemistry of iron and steel manufacture. 2. ed. London: Longman, 1972. p. 193-484.

6 RASSBACH, T.; SAUDERES, E.R. Reducing period in stainless steel melting. Journal of Metals, v. 8, p. 1009-1015, 1953.

7 NOLASCO SOBRINHO, P.J.; ESPINOSA, D.C.R.; TENÓRIO, J.A.S. Characterisation of dusts and sludges generated during stainless steel production in Brazilian industries. Ironmaking and Steelmaking, v.30, n. I, p.I I-17, 2003.

8 NOLASCO SOBRINHO, P.j.; ESPINOSA,D.C.R.; TENÓRIO, J.A.S. Estudo morfológico da poeira gerada na fabricação de aço inoxidável. In: Seminário de Fusão, Refino e Solidificação de Metais, 31., 2000, Vitória. Anais... São Paulo: ABM, 2000. v. I, p.473-479.

9 NOLASCO-SOBRINHO, P.j.; ESPINOSA, D.C.R.; TENÓRIO, J.A.S. Caracterização de poeira gerada na aciaria durante a fabricação de aço inoxidável. In: Seminário de Fusão, Refino e Solidificação de Metais, 31., 2000, Vitória. Anais... São Paulo: ABM, 2000. v. I, p.465-472.

10 GÖRNERUP, M.; LAHIRI, K . Reduction of electric arc furnace slags in stainless steelmaking. Part 2: Mechanism of CrOx reduction. ISIJ International, v. 25, n.5, p. 382-386, 1998.

II TSOMONDO, M.B.C; SIMBI, D.J.; NAVARA, E. Isothermal reduction of chromite fines by carbon saturated chromium melt. Ironmaking and Steelmaking, v.24, n.5, p. 386-39I, 1997.

I2 NAKASUGA, T.; SUN, H.P.; NAKASHIMA, K.; MORI, K. Reduction rate of $\mathrm{Cr} 2 \mathrm{O} 3$ a solid powder state and in $\mathrm{CaO}-$ SiO2-AI2O3-CaF2 slags by Fe-C-Si melts. ISIJ International, v. 4I, n. 9, p. 937-944, 200 I.

13 Kundrat, D.M.; SMILLIE; A.M.; SUSSMAN; R.C. Method for direct use of chromite ore in the production of stainless steel. US Patent 5.702.502. 1997.

14 NOLASCO SOBRINHO, P.J. Reciclagem de poeiras e lamas provenientes da fabricação de aço inoxidável. 2004. 144p. Tese (Doutorado em Engenharia. Área: Engenharia Metalúrgica e de Materiais) - Escola Politécnica da Universidade de São Paulo(USP). São Paulo, 2004.

Recebido em: 22/10/07

Aceito em: 14/02/08

Proveniente de: SEMINÁRIO DE FUSÃO, REFINO E SOLIDIFICAÇÃO DOS METAIS - INTERNACIONAL, 36., 2005, Vitória, ES. São Paulo: ABM, 2005. 